\title{
Angry Birds Realized: Water Balloon Launcher for Teaching Projectile Motion With Drag
}

\author{
Boyd F. Edwards, ${ }^{*}$ David D. Sam, Michael A. Christiansen, William A. Booth, and Leslie O. Jessup \\ Utah State University Uintah Basin \\ Vernal, UT 84078 \\ (Dated: March 14, 2014)
}

\begin{abstract}
A simple, collapsible design for a large water balloon slingshot launcher features a fully adjustable initial velocity vector and a balanced launch platform. The design facilitates quantitative explorations of the dependence of the balloon range and time of flight on the initial speed, launch angle, and projectile mass, in an environment where quadratic air drag is important. Presented are theory and experiments that characterize this drag, and theory and experiments that characterize the nonlinear elastic energy and hysteresis of the latex tubing used in the slingshot. The experiments can be carried out with inexpensive and readily available tools and materials. The launcher provides an engaging way to teach projectile motion and elastic energy to students of a wide variety of ages.
\end{abstract}

\section{INTRODUCTION}

One goal of the Summer 2012 Science and Engineering Camp held at Utah State University - Uintah Basin was to capitalize on the popularity of the Angry Birds video game to excite seventh and eighth grade students about projectile motion and quantitative science. To this end, we designed a slingshot device capable of lobbing a small water balloon a distance of $85 \mathrm{~m}$, with fully adjustable and quantifiable launch angle and initial slingshot displacement [1]. The device inspired a television news story [2] that was aired by $53 \mathrm{NBC}$ affiliate stations across 29 states in the United States and has already been replicated by at least one individual $[3,4]$. The purpose of the present paper is to introduce the device as a tool for teaching projectile motion, air drag, and elastic energy.

In Angry Birds, players use a simulated slingshot to lob wingless birds at pigs stationed on various structures, aiming the birds by adjusting their initial displacements in the slingshot. The video game has been downloaded over 1.7 billion times [5] and has been called "the largest mobile app success the world has seen so far" [6].

Our realization of the Angry Birds concept is a slingshot device that uses biodegradable water balloons as projectiles to enable students to easily identify landing sites, where balloons burst and dampen the ground. Balloons also enable investigations of the consequences of changing the projectile mass $m$ simply by changing the mass of water in the balloons. In contrast with previous water balloon launcher designs [7-12], our design allows students to precisely specify a full range of initial slingshot displacements and launch angles.

The design is simple, inexpensive to build, and collapsible (Fig. 1). The horizontal pivot axis (A) of the launch platform passes through its center of mass, enabling effortless movement of the platform to an elevation angle $\phi$ and ensuring, through joint friction, that the platform remain at this angle. This axis is located at a height $h$

\footnotetext{
*boyd.edwards@usu.edu
}

above the ground. The platform angle $\phi$ ranges from $0^{\circ}$ (horizontal) to $90^{\circ}$ (vertical), and is read directly from an angle finder (B) attached to the platform. A slingshot includes latex tubing, two handles that attach to the platform arms at $\mathrm{C}$ and $\mathrm{C}^{\prime}$, and a balloon pouch (D) that attaches to the platform by a trigger device $(\mathrm{E})$. The handle attachment points $\left(\mathrm{C}\right.$ and $\left.\mathrm{C}^{\prime}\right)$ are adjustable to allow for variations in the initial slingshot displacement $b$ (Fig. 2). A syringe-like "Water Blast" tube is used to quickly fill water balloons with a specified volume of water, yielding balloon masses $m$ that are reproducible to within $1 \%$. Detailed parts lists, specifications, construction plans, and operating procedures are discussed elsewhere [1].

At the summer camp, the Angry Birds workshop was presented to groups of 15 students, ages 13 and 14. Each workshop lasted 80 minutes and was divided approximately into a 20-minute classroom introduction, a 40minute outdoor experiment, and a 20-minute classroom conclusion. In the introduction, the students were divided into three teams of five and were issued safety goggles. Each team was assigned an experiment and an adult facilitator to assist with the experiment and to ensure that goggles were properly worn. Goggles protect against potential breakage of stretched latex tubing and against accidental impact with flying water balloons, which can reach speeds over $35 \mathrm{~m} / \mathrm{s}$.

Experiment 1 was to measure the horizontal balloon range $R$ vs. $\phi$, with $m$ and $b$ constant. Experiment 2 was to measure $R$ vs. $b$, with $m$ and $\phi$ constant. Experiment 3 was to measure $R$ vs. $m$, with $\phi$ and $b$ constant. In the classroom introduction, the students were asked to predict the outcomes of the three experiments. For the outdoor experiments, three launcher devices (one per team) were lined up side-by-side at one end of a $100 \mathrm{~m}$ field marked by labeled flags inserted into the ground every 5 $\mathrm{m}$. The students used meter sticks to interpolate $R$ to the nearest meter, and recorded and plotted their results on worksheets [1]. Each team made seven measurements, using water balloons that had been filled previously by facilitators. In the classroom conclusion, a captain chosen from each team presented and explained the team's 

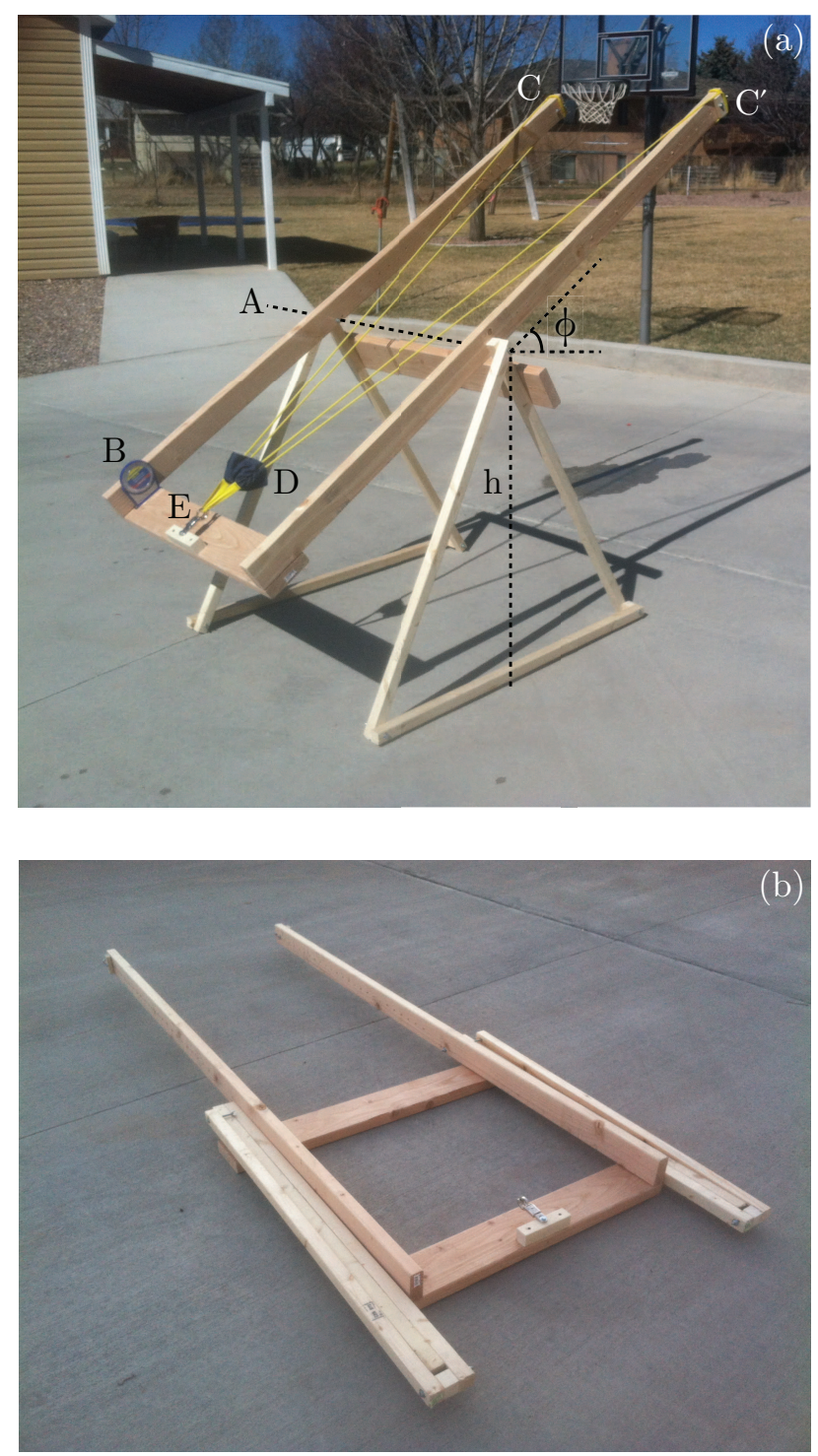

FIG. 1. Photographs of the balloon launcher device assembled (a) and collapsed (b). The launch platform pivots about a horizontal axis A. The elevation angle $\phi$ of the platform can be read directly from an angle finder B. Four bands of latex tubing attach to the platform at movable points $\mathrm{C}$ and $\mathrm{C}^{\prime}$, and to a pouch $\mathrm{D}$, which attaches to a trigger E. Water balloons are launched by inserting them into the pouch and by releasing the trigger.

results to the other teams.

An instructive and entertaining outdoor exercise, one that closely mimics the spirit of Angry Birds, is to ask an adult target (the "pig") to stand at a specified distance from the launchers (40 m, say), and to allow each team to take one shot at him. We did this exercise after the teams plotted their results, which they used to select $\phi$, $m$, and $b$ for their attempts. Featured as pigs were Utah Senator Kevin Van Tassell and Geoff Liesik, the reporter for the television news story, which included footage of his being splattered by a water balloon [2].

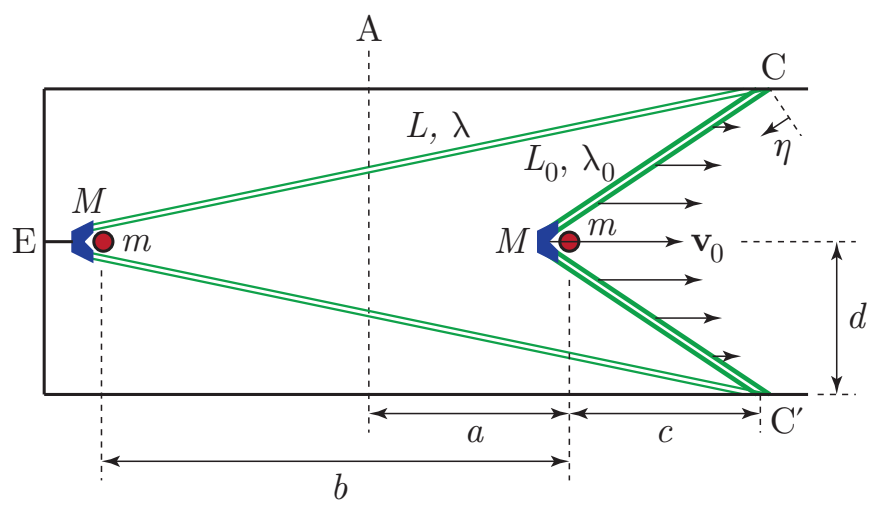

FIG. 2. Schematic of the launch platform. Two bands of latex surgical tubing, each of natural length $L_{0}$ and linear mass density $\lambda_{0}$, attach to the platform at $\mathrm{C}$, and two similar bands attach at $\mathrm{C}^{\prime}$. The four bands meet at a pouch of mass $M$ that holds a projectile of mass $m$. Displacing the pouch a distance $b$ and attaching it to a trigger at E stretches each band to length $L$ and linear mass density $\lambda=\lambda_{0} L_{0} / L$. After releasing the trigger, the bands return to their natural lengths and the projectile is released by the slingshot with velocity $\mathbf{v}_{0}$. Because $L_{0}$ is larger than the platform half width $d$, the slingshot releases the projectile before it passes the line joining $\mathrm{C}$ and $\mathrm{C}^{\prime}$. Shown also are the platform pivot axis $\mathrm{A}$, the distance $a$ from this axis to the launch point, and the distance $c$ from this point to the line joining $\mathrm{C}$ and $\mathrm{C}^{\prime}$.

Besides providing an engaging way to teach basic concepts of projectile motion to children, the device has much to teach physics undergraduate students about air drag and nonlinear elastic energy. Despite the familiarity of the concept of air drag to undergraduate students in principle, they have neglected it so many times in practice that they may develop a fear that it is "hard."

It is not. Reviewed below to elucidate such popular concepts as speed decay and terminal speed are simple rectilinear closed-form solutions for quadratic drag, that is, a drag force that is proportional to the square of the speed $v$. While linear "Stokes" drag applies for small velocities and microscopic projectiles, quadratic drag applies for the conditions of interest, namely, macroscopic projectiles with speeds $v$ in the range $20-40 \mathrm{~m} / \mathrm{s}$, radii $r$ in the range $2-5 \mathrm{~cm}$, and Reynolds numbers $\mathrm{Re}=v \mathrm{r} / \nu$ in the range $5-7 \times 10^{4}$, where $\nu=1.6 \times 10^{-5} \mathrm{~m}^{2} / \mathrm{s}$ is the kinematic viscosity of air [13-18]. The range for Re is small because the fixed potential energy of the stretched bands implies that $v$ decreases with increasing $m$ and $r$, whence the low end of the speed range corresponds to the high end of the radius range. We restrict our attention to quadratic drag, which offers rectilinear solutions that are easily accessible to undergraduate students.

To enable comparison of our measurements of the time of flight for vertical launches with theory, we generalize the calculations of Ref. [19] for vertical projectile motion with quadratic drag to account for unequal initial and final heights. Because theoretical results for nearhorizontal motion [20] and two-dimensional motion [2123 ] may exceed the reach of physics undergraduate stu- 
dents, we obtain two-dimensional solutions by simple Euler integration.

Projectile motion with air resistance has received a great deal of attention in the literature. Drag cannot reasonably be neglected in many outdoor sports and other applications [24-28]. In addition to drag, spinning balls experience a Magnus force that is perpendicular to the direction of motion, such as the lift force on a ball with backspin [29-31]. Measuring spin requires high-speed photography and careful triangulation [29]. We ignore any spin of our water balloons, assuming that the contact of the slingshot pouch with the balloons and the equal lengths of the four slingshot bands impart negligible spin on balloons as they are launched. Likewise, we have made no attempt to observe any deformations or vibrations of our flexible water balloons during flight. Others have examined the effect of asymmetry and roughness of the ball on its motion [32].

Many different launching methods have been used over the years to study projectile motion, including curved tubes [33], projectiles released from an object moving horizontally [34], lacrosse sticks [32], tennis ball launchers [27], baseball pitching machines [29], soccer ball launchers [31], and hand throwing [35, 36]. Some have even used an Xbox Kinect sensor device to measure projectile motion [37]. Advantages of our slingshot design include the reproducibility of the initial projectile velocity and the full range and ease of its adjustment.

Attention has been devoted to the launch angle $\phi_{\max }$ that maximizes $R$ for projectile motion of a spinless ball with equal initial and final heights. Without drag, $\phi_{\max }=45^{\circ}$. Linear and quadratic drag imply $\phi_{\max }<$ $45^{\circ}$, while strong drag forces proportional to $v^{n}$ with $n>2$ sometimes yield $\phi_{\max }>45^{\circ}[38-40]$.

For low-density projectiles such as beach balls, the drag depends on the projectile acceleration [36]. This effect is negligible for soccer balls [31, 41, 42], and we ignore it for our high-density water balloons.

The small but observable energy losses of the stretched latex tubing used in the slingshot offer an example of nonlinear elastic energy and hysteresis in an environment where energy losses can ultimately be neglected, and offer an opportunity to review what it really means for a force to be conservative. Elastic hysteresis is particularly accessible to undergraduate students, and may help to prepare them for the more complicated processes of ferromagnetic and ferroelectric hysteresis. Elastic hysteresis has been studied previously for torsion of rubber tubing [43], stretched bands [44, 45], bent plastic foil [46], and compressed balls and Silly Putty [47-49].

In Sec. II, we report measurements of the nonlinear elastic force of stretched latex tubing and its small elastic hysteresis and energy losses. We justify the neglect of these losses and introduce an associated elastic potential. We introduce simple closed empirical forms that accurately represent the nonlinear elastic force and elastic potential. In Sec. III, we use this potential and conservation of energy, including the inertia of the slingshot bands and pouch, to predict the launch speed vs. projectile mass and compare this prediction favorably with measurements. In Sec. IV, we review Newton's second law applied to a spinless projectile subject to both quadratic drag and a uniform gravitational field, and solve this equation in instructive one-dimensional cases, including a vertical launch with unequal initial and final heights. We determine the average drag coefficient of water balloons for vertical launches using time-of-flight measurements. In Sec. V, we use this coefficient to compare measurements of the horizontal range with predictions obtained by Euler integration.

\section{ELASTIC ENERGY}

Four bands of latex tubing supply the energy of the launch, each band with natural length $L_{0}=79 \mathrm{~cm}$ and linear mass density $\lambda_{0}=0.0427 \mathrm{~kg} / \mathrm{m}$. These bands are stretched to a length $L$ that depends on the band attachment locations ( $\mathrm{C}$ and $\mathrm{C}^{\prime}$ in Fig. 2). To determine the elastic properties of this tubing, we suspend a single band of length $L_{0}=79 \mathrm{~cm}$ and measure its downward extension $x=L-L_{0}$ for different attached masses $m$. Applying Newton's second law gives the $x$-component of the equilibrium elastic force, $F_{x}=-m g$, where $g=9.8$ $\mathrm{m} / \mathrm{s}^{2}$ is the magnitude of the gravitational acceleration. Increasing $m$ by adding 205-gram masses one-by-one produces the "loading" force of Fig. 3 (squares and solid trace), with maximum extension $X=131 \mathrm{~cm}$. Removing these masses one-by-one produces the "unloading" force (circles and solid trace).

The loading and unloading forces differ slightly. To characterize this elastic hysteresis, we consider the work done by the elastic force,

$$
W_{x_{1} \rightarrow x_{2}}=\int_{x_{1}}^{x_{2}} F_{x}(x) d x
$$

represented in Fig. 3 as the area under the trace. The elastic force is nonconservative because the work it does in a closed loop is nonzero;

$$
W_{0 \rightarrow X}+W_{X \rightarrow 0}=-E,
$$

where $E>0$ represents the both energy lost to internal friction during the loading / unloading cycle and the area of the hysteresis loop in Fig. 3. Integrating numerically with linear interpolation between data points yields $W_{0 \rightarrow X}=-33.9 \mathrm{~J}$, the work done by the elastic force during loading, for which the elastic force is antiparallel to the displacement, and $W_{X \rightarrow 0}=33.0 \mathrm{~J}$, the work done by the elastic force during unloading, for which the elastic force is parallel to the displacement. Equation (2) gives the associated energy loss, $E=0.9 \mathrm{~J}$.

Because $E \ll W_{X \rightarrow 0}$, we neglect internal friction and define an elastic potential energy $U(x)$ by $F_{x}=-d U / d x$. Since the projectile is launched by unloading the bands, 


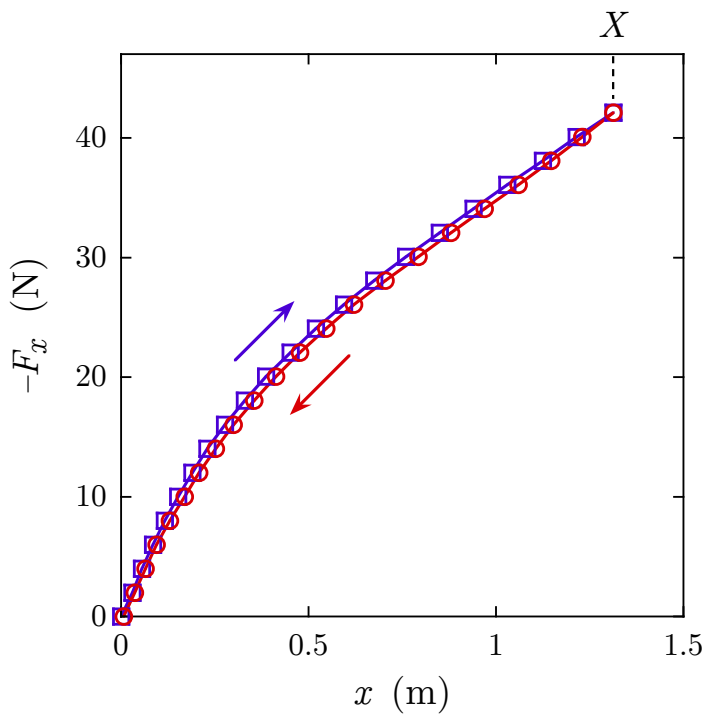

FIG. 3. Measured nonlinear elastic force $F_{x}$ in Newtons vs. extension $x$ in meters for a single band of elastic tubing of length $79 \mathrm{~cm}$ during loading (squares, solid trace) and unloading (circles, solid trace), for a maximum band extension of $X=1.31$ $\mathrm{m}$. The area between the two traces represents the energy lost to friction during the loading and unloading cycle.

we use the unloading force to numerically integrate

$$
U(x)=-\int_{0}^{x} F_{x^{\prime}}\left(x^{\prime}\right) d x^{\prime}
$$

using linear interpolation between data points.

Resulting numerical values of $U$ are shown in Fig. 4 (diamonds) together with the measurements of the unloading force $F_{x}$ from Fig. 3 (circles). Also shown in Fig. 4 are the empirical approximations (solid traces)

$$
F_{x}(x)=-k x-A\left(1-e^{-\alpha x}\right)
$$

and

$$
U(x)=\frac{1}{2} k x^{2}+A x-\frac{A}{\alpha}\left(1-e^{-\alpha x}\right),
$$

with $k=22.68 \mathrm{~N} / \mathrm{m}, A=12.08 \mathrm{~N}$, and $\alpha=4.79 \mathrm{~m}^{-1}$ chosen to minimize the sum of the squares of the differences between the numerical results for $U$ and Eq. (5). Indeed, while Eq. (4) agrees with our measurements of $F_{x}$ to within $1 \mathrm{~N}$, Eq. (5) agrees with our numerical results for $U$ to within $0.1 \mathrm{~J}$. Here, $k$ represents the elastic force constant that governs the linear Hooke's law behavior of $F_{x}$ for large values of $x$ (dashed trace in Fig. 4), and $A$ and $\alpha$ govern the nonlinear behavior for small $x$. Note that the specific values of $k, A$, and $\alpha$ likely depend on the length $L_{0}$ of the band. Equation (4) matches our experimental data better than a state equation used to study the elastic behavior of rubber bands [44].

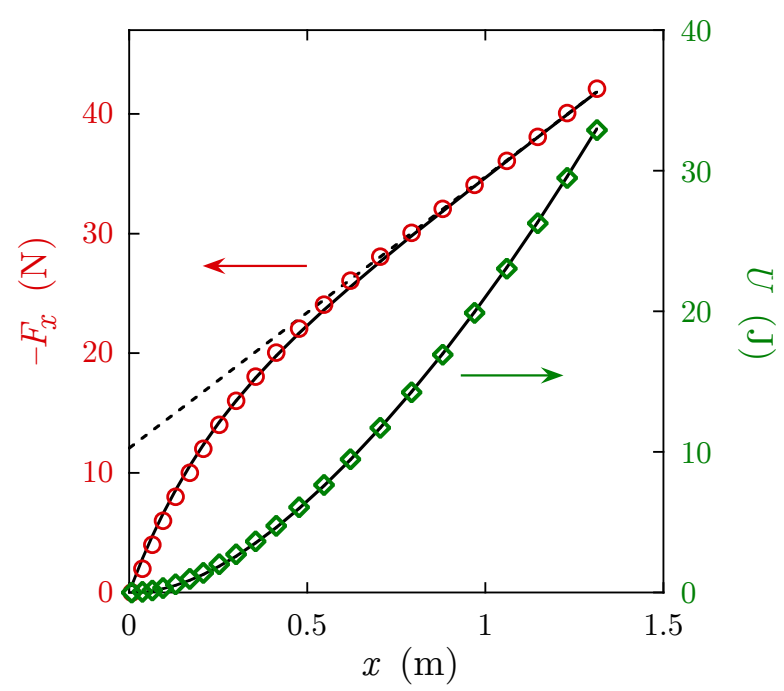

FIG. 4. Measured elastic unloading force $F_{x}$ in Newtons (circles, from Fig. 3) and corresponding elastic potential $U$ in Joules obtained by numerical integration of Eq. (3) (diamonds) vs. extension $x$ in meters for a single band of elastic tubing of length $79 \mathrm{~cm}$. Solid traces are empirical approximations given by Eqs. (4) and (5), and the dashed trace gives the asymptotic linear behavior of Eq. (4).

\section{INITIAL SPEED}

We use conservation of energy, with Eq. (5) describing the elastic potential, to determine the speed $v_{0}$ of the projectile as it leaves the slingshot. We consider the time between the release of the trigger, when the bands, pouch, and projectile are momentarily at rest with the bands stretched to length $L$, and the launch point, where the bands reach their natural length $L_{0}$ and the slingshot releases the projectile with speed $v_{0}$ (Fig. 2). We ignore air drag during the launch but include the masses of the pouch and bands, which play a significant role for projectile masses of interest.

To simplify the analysis, we neglect gravitational deflection of the projectile during the launch by assuming that the projectile executes rectilinear motion in the plane of the launch platform (Fig. 2). When the projectile is released by the trigger, the net elastic force of the four bands overwhelms the gravitational force on the projectile. However, as a projectile approaches its launch point, the elastic force decreases to zero, giving gravity an opportunity to deflect the projectile for non-vertical launches. Measurements reported in Sec. V indicate that this deflection may be observable for small launch angles.

Conservation of energy gives

$$
\frac{1}{2} m_{k} v_{0}^{2}+m_{p} g b \sin \phi=4 U(X)
$$


where

$$
m_{k}=m+M+\frac{4}{3} \lambda_{0} L_{0}
$$

accounts for the kinetic energy of the projectile of mass $m$, the pouch of mass $M$, and the bands of linear mass density $\lambda_{0}$,

$$
m_{p}=m+M+2 \lambda_{0} L_{0}
$$

accounts for the change in gravitational potential energy of the projectile, the pouch, and the bands, and

$$
U(X)=\frac{1}{2} k X^{2}+A X-\frac{A}{\alpha}\left(1-e^{-\alpha X}\right)
$$

gives the elastic potential energy of one of the four stretched bands, with extension $X=L-L_{0}$, from Eq. (5).

To calculate the kinetic energy of the bands at launch, we define $\eta$ to be the distance from the attachment point to a tubing element of mass $\lambda_{0} d \eta$ and assume that the speed $v_{0} \eta / L_{0}$ of this element is proportional to $\eta$, giving a speed of zero at the attachment point and a speed of $v_{0}$ at $\eta=L_{0}$ (Fig. 2). Thus the kinetic energy of this element is $d K=(1 / 2)\left(\lambda_{0} d \eta\right)\left(v_{0} \eta / L_{0}\right)^{2}$. Integrating yields the kinetic energy of one band,

$$
K=\int_{0}^{L_{0}} d K=\frac{\lambda_{0} L_{0}}{6} v_{0}^{2} .
$$

Multiplying this result by 4 (for the four bands of tubing) gives the contribution of the bands to the kinetic energy appearing in Eqs. (6) and (7).

The change in the gravitational potential energy of the bands is given by $4 \lambda_{0} L_{0} g \Delta y$, where $4 \lambda_{0} L_{0}$ is the mass of the four bands, $g$ is the magnitude of the gravitational acceleration, and $\Delta y$ is the change in the height of the center of mass of the bands. During the launch, this center of mass moves a distance $b / 2$ along the launch platform (Fig. 2) and rises a vertical distance $\Delta y=(b / 2) \sin \phi$, yielding $2 \lambda_{0} L_{0} g b \sin \phi$ as the change in gravitational potential energy of the bands appearing in Eqs. (6) and (8).

To test Eq. (6), we measured $v_{0}$ for a series of horizontal launches with balloon masses in the practical range $50 \mathrm{~g}<m<600 \mathrm{~g}$. Setting $\phi=0$ and rearranging yields the predicted behavior

$$
v_{0}(m)=2 \sqrt{\frac{2 U(X)}{m^{\prime}+m}},
$$

where $m^{\prime}=M+4 \lambda_{0} L_{0} / 3$. The measurements were carried out for $L_{0}=79 \mathrm{~cm}, L=193 \mathrm{~cm}, X=114 \mathrm{~cm}$, $M=0.02571 \mathrm{~kg}$, and $\lambda_{0}=0.0427 \mathrm{~kg} / \mathrm{m}$, which yield $m^{\prime}=0.0707 \mathrm{~kg}$ and $U(X)=26.0 \mathrm{~J}$ from Eq. (9).

For each measurement of $v_{0}$, two water balloons were prepared with masses within $1 \mathrm{~g}$ of each other. One of these was launched horizontally at a vertical wooden target located $3 \mathrm{~m}$ from the launch point and resting on a table. The other was launched at the same target, now located $4 \mathrm{~m}$ from the launch point on the same table. In each case, the time between the impact of the trigger latch with a steel pipe and the impact of the balloon with the target was determined from an audio recording with a sampling rate of $48 \mathrm{kHz}$, made using the iPhone software application TwistedWave. The difference $\Delta t$ between these times is the time required for the projectile to travel a distance of $\Delta x=1 \mathrm{~m}$, which yield $v_{0}=\Delta x / \Delta t$. The main source of error in the measurements is the uncertainty in the time of impact of the trigger latch, which implies an uncertainty of about $\delta t=0.001 \mathrm{~s}$ in $\Delta t$ and an uncertainty of $v_{0} \delta t / \Delta t$ in $v_{0}$. The relative uncertainty $\delta t / \Delta t$ in the time exceeds the relative uncertainty in the balloon mass, which helps to justify our assumption that the two balloons used in each measurement are launched at the same speed.

The impact times (of the trigger latch with the pipe, and of the balloon with the target) were designated as the instants at which their recorded sound pressure first reached or exceeded the maximum pressure of the recording software, saturating the signal. These impact times were determined by zooming in on the audio waveform using TwistedWave. The uncertainty in these measurements is the difference between the times at which the maximum and half-maximum pressures were reached.

The iPhone was placed an equal distance from the two target positions, at a distance of about $3 \mathrm{~m}$, to avoid the need to account for the speed of sound. The iPhone was placed with an unimpeded line of sight between it and each target position, and between it and the trigger, so it could receive the direct sound from each, not delayed reflections. To minimize such reflections, the measurements were taken outside, away from reflective surfaces. The launcher supports were weighted to ensure that it remained fixed.

Our measurements of $v_{0}$ (open circles in Fig. 5) agree well with Eq. (11) (solid trace) except for the two smallest balloons. Correcting for air drag during these short (3 m) horizontal flights (Sec. IV) improves this agreement (filled circles in Fig. 5, with uncertainties shown with error bars). Agreement between theoretical and experimental values of $v_{0}$ confirms our neglect of elastic energy losses, the form of our elastic potential, and our treatment of the kinetic energy of the bands and pouch. Also shown in Fig. 5 are corresponding values of $r$ given by Eq. (15).

\section{DRAG COEFFICIENT}

To assess the importance of air drag in the waterballoon trajectories achievable by the launcher and to determine the drag coefficient of these balloons, we consider Newton's second law with gravitational and quadratic drag forces. We assume that the balloons are spherical, of radius $r$, mass $m$, velocity $\mathbf{v}$, drag coefficient $C$, and cross-sectional area $A=\pi r^{2}$ moving without spin 


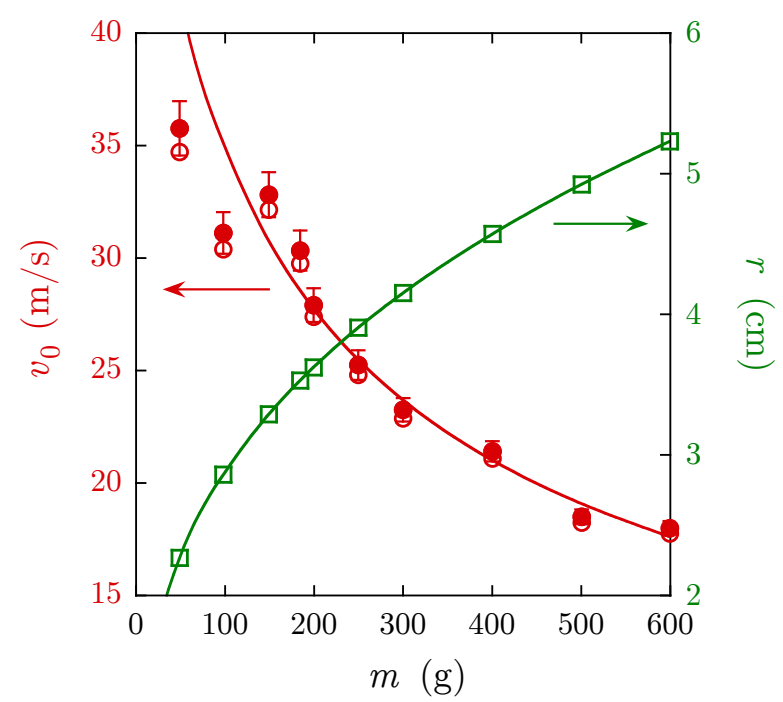

FIG. 5. Measured horizontal launch speeds $v_{0}$ in meters per second vs. balloon mass $m$ in grams before correcting for drag (open circles) and after correcting for drag (filled circles), compared with Eq. (11) (solid trace). Also shown (squares and solid trace) are the corresponding balloon radii $r$ in $\mathrm{cm}$ from Eq. (15).

through still air of mass density $\rho_{a}$ in the presence of a uniform gravitational field $\mathbf{g}$, writing [51]

$$
m \dot{\mathbf{v}}=m \mathbf{g}-\frac{1}{2} \rho_{a} C A v \mathbf{v} .
$$

Ignoring the volume and the mass (less than $1 \mathrm{~g}$ ) of the balloon membrane, writing $V=(4 / 3) \pi r^{3}$ as the volume of the water in the balloon, and writing $\rho_{w}=m / V$ as the mass density of water allows us to rewrite Eq. (12) as

$$
\dot{\mathbf{v}}=\mathbf{g}-\kappa v \mathbf{v}
$$

The drag constant

$$
\kappa=\frac{3}{8} \frac{\rho_{a}}{\rho_{w}} \frac{C}{r}
$$

has units of inverse meters and has a mass-dependent radius

$$
r=\left(\frac{3 m}{4 \pi \rho_{w}}\right)^{1 / 3} .
$$

Buoyancy is negligible because $\rho_{a} / \rho_{w} \ll 1$.

Rectilinear motion in the absence of gravity reveals the physical interpretation of $\kappa$. Substituting $\mathbf{g}=0$ and $\mathbf{v}=v(x(t)) \hat{x}$ into Eq. (13), applying the chain rule, and integrating yields

$$
v(x)=v_{0} e^{-\kappa\left(x-x_{0}\right)},
$$

where the coordinate $x$ is in the direction of motion, $x_{0}$ is the initial position, and $v_{0}$ is the initial speed. Thus, in the absence of other forces, quadratic air drag produces an exponential spatial decay of the speed, with $\kappa^{-1}$ representing the $1 / e$ decay distance. Because $\kappa \propto 1 / r$, this decay distance increases, and the importance of drag decreases, with increasing projectile size.

The drag coefficient $C$ depends on the Reynolds number $\operatorname{Re}=v r / \nu$. Values of $v_{0}$ and $r$ shown in Fig. 5 together with the kinematic viscosity of air, $\nu=1.6 \times 10^{-5}$ $\mathrm{m}^{2} / \mathrm{s}$ [18], yield values of Re in the range $5.1-6.7 \times 10^{4}$. These values fall in the range $3 \times 10^{2}<\operatorname{Re}<1.3 \times 10^{5}$ for high-Reynolds-number flow that is laminar on the windward side of the projectile and turbulent in its wake, with $C \approx 0.5$ in this range for smooth spherical projectiles [19, 50-52]. When the Reynolds number exceeds the upper limit of this range, the drag coefficient drops precipitously to $C \approx 0.1$ at the "drag crisis."

To determine $C$ for water balloons, which are neither completely smooth nor spherical, we compare calculations and measurements of the time of flight for vertical launches. Taking the $y$ axis as vertically upward with $\mathbf{g}=-g \hat{y}$ and $\mathbf{v}=v_{y} \hat{y}$, we use Eq. (13) to calculate the time $t_{1}$ required for a projectile at initial height $y_{0}$ and initial velocity $\mathbf{v}_{0}=v_{0} \hat{y}$ to ascend to its apex at $y=Y$, where its velocity vanishes. We then calculate the time $t_{2}$ required for the projectile to descend from the apex to the ground at $y=0$, and determine the total time of flight from $T=t_{1}+t_{2}$. We assume for simplicity that $\kappa$ and $C$ are constants that apply throughout the duration of the flight.

For the ascent with $v_{y}>0$, the velocity is antiparallel to both gravity and drag, $v=v_{y}$, and Eq. (13) becomes

$$
\dot{v}_{y}=-g-\kappa v_{y}^{2}
$$

Integrating with respect to time and demanding $v_{y}=0$ at the apex yields the time to the apex,

$$
t_{1}=\tau \tan ^{-1}\left(v_{0} / v_{t}\right)
$$

where

$$
v_{t}=\sqrt{\frac{g}{\kappa}}
$$

is the terminal speed and

$$
\tau=\frac{1}{\sqrt{\kappa g}}
$$

is the characteristic time of approach to this speed. A second integration yields the height of the apex,

$$
Y=y_{0}+\frac{1}{2 \kappa} \ln \left(1+\frac{v_{0}^{2}}{v_{t}^{2}}\right)
$$

For the descent with $v_{y}<0$, the velocity is parallel to gravity and antiparallel to drag, $v=-v_{y}$, and Eq. (13) becomes

$$
\dot{v}_{y}=-g+\kappa v_{y}^{2}
$$


Integrating with $t=0$ at the apex yields the timedependent velocity [53]

$$
v_{y}(t)=-v_{t} \tanh (t / \tau)
$$

and acceleration

$$
\dot{v}_{y}(t)=-g \operatorname{sech}^{2}(t / \tau) .
$$

These results confirm the physical interpretations of $v_{t}$ and $\tau$ given above. The speed of a particle that is released from rest in a uniform gravitational field increases asymptotically to the terminal speed $v_{t}$ in a characteristic time $\tau$, while the magnitude of its acceleration decreases asymptotically from $g$ to zero. Integrating Eq. (23) yields the descent time,

$$
t_{2}=\tau \cosh ^{-1} e^{\kappa Y} .
$$

Combining Eqs. (18), (21), and (25) yields the total time of flight,

$$
T=\tau \tan ^{-1}\left(v_{0} / v_{t}\right)+\tau \cosh ^{-1}\left(e^{\kappa y_{0}} \sqrt{1+v_{0}^{2} / v_{t}^{2}}\right) .
$$

Equations (21) and (26) give the maximum height and the total time of flight for vertical trajectories with unequal initial and final heights. Setting $y_{0}=0$ yields Eqs. (33) and (44) of Ref. [19] for equal initial and final heights.

The initial speed

$$
v_{0}=\left[\frac{8 U(X)}{m_{k}}-2 \frac{m_{p}}{m_{k}} g b\right]^{1 / 2}
$$

follows from Eq. (6) with $\phi=90^{\circ}$, with $m_{k}, m_{p}$, and $U(X)$ given by Eqs. (7), (8), and (9). The increase in gravitational potential energy during this vertical launch renders its initial speed 1-4\% smaller than the horizontal launch speed given by Eq. (11) and shown in Fig. 5. The initial height is (Figs. 1 and 2)

$$
y_{0}=h+a .
$$

In the limit $\kappa \rightarrow 0$, Eqs. (21) and (26) reduce to the elementary results

$$
Y=y_{0}+\frac{v_{0}^{2}}{2 g}
$$

and

$$
T=\frac{v_{0}}{g}+\frac{1}{g} \sqrt{v_{0}^{2}+2 g y_{0}}
$$

for projectile motion with constant gravitational acceleration and no drag.

The drag constant $\kappa$ increases with decreasing mass $m$ according to $\kappa \propto m^{-1 / 3}$ [Eq. (14), Eq. (15), and Fig. 6], implying that drag is important for small particles. The corresponding terminal speed $v_{t} \propto m^{1 / 6}$ and decay time

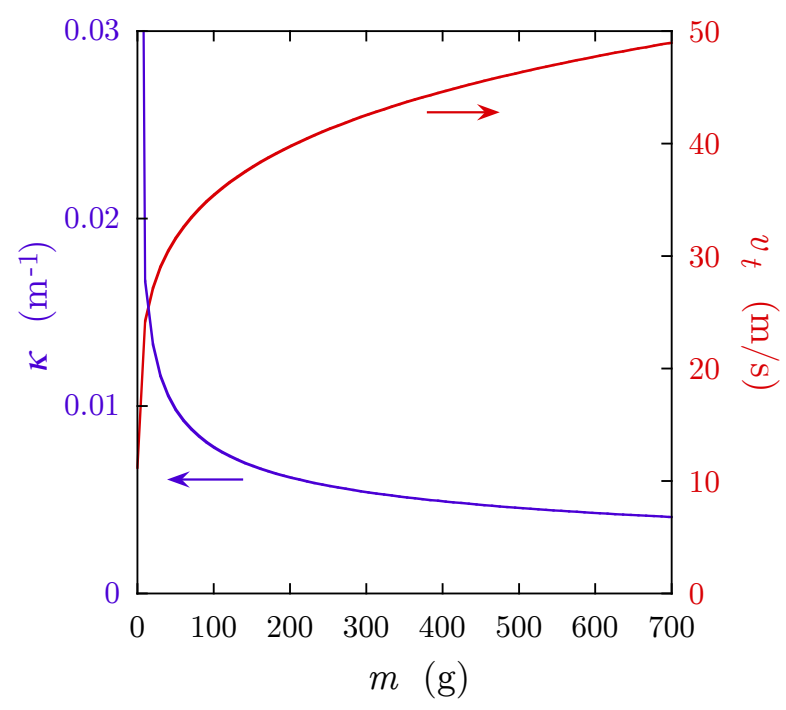

FIG. 6. Shown are the drag constant $\kappa$ in inverse meters and the terminal speed $v_{t}=(g / \kappa)^{1 / 2}$ in meters per second vs. balloon mass $m$ in grams, from Eqs. (14), (15), and (19).

$\tau \propto m^{1 / 6}$ both vanish as $m \rightarrow 0$ [Eq. (19), Eq. (20), and Fig. 6], implying suspension of vanishingly small particles. Indeed, small water droplets and ice crystals are suspended in clouds until the particles accumulate enough mass to begin falling to the earth [54].

We measured the time of flight $T=T_{t}-T_{l}$ for vertical launches by using TwistedWave to determine the total time $T_{t}$ between the impact of the launcher trigger latch with a steel pipe and the impact of the balloon with the ground, and subtracting the launch time $T_{l}$, the time between the impact of the trigger latch and the release of the projectile by the slingshot. For simplicity, we estimate the launch time $T_{l}=b / v_{0}$ to be the ratio of the launch distance $b$ to the initial speed $v_{0}$, given that projectile acceleration is largest during the early stages of the launch when the net elastic force is greatest, and given the smallness of $T_{l}<0.02 T_{t}$. We used the values $C=0.55, \rho_{a}=1.2 \mathrm{~kg} / \mathrm{m}^{3}, \rho_{w}=1000 \mathrm{~kg} / \mathrm{m}^{3}, a=0.56$ $\mathrm{m}, b=1.23 \mathrm{~m}, h=1.05 \mathrm{~m}, y_{0}=1.61 \mathrm{~m}, g=9.8 \mathrm{~m} / \mathrm{s}^{2}$, $L_{0}=79 \mathrm{~cm}, L=193 \mathrm{~cm}, X=114 \mathrm{~cm}, M=0.02571$ $\mathrm{kg}$, and $\lambda_{0}=0.0427 \mathrm{~kg} / \mathrm{m}$, whence $U(X)=26.0 \mathrm{~J}$ from Eq. (9).

Figure 7 shows the agreement between these measurements of $T$ and Eq. (26), with $v_{0}$ given by Eq. (27). This agreement indicates that water balloons have drag coefficients $C=0.55 \pm 0.05$ that might slightly exceed the value $C \approx 0.5$ for smooth spheres $[50,51]$. The value $C=0.55 \pm 0.05$ fits both the time-of-flight measurements shown in Fig. 7, which tend to favor slightly smaller values of $C$, and the large- $\phi$ range measurements in Fig. 8, which tend to favor slightly larger values. Since $C$ varies with Re, and since Re varies with time while a balloon is in flight, the value $C=0.55$ should be considered as an average value, one that does a reasonable job for the 


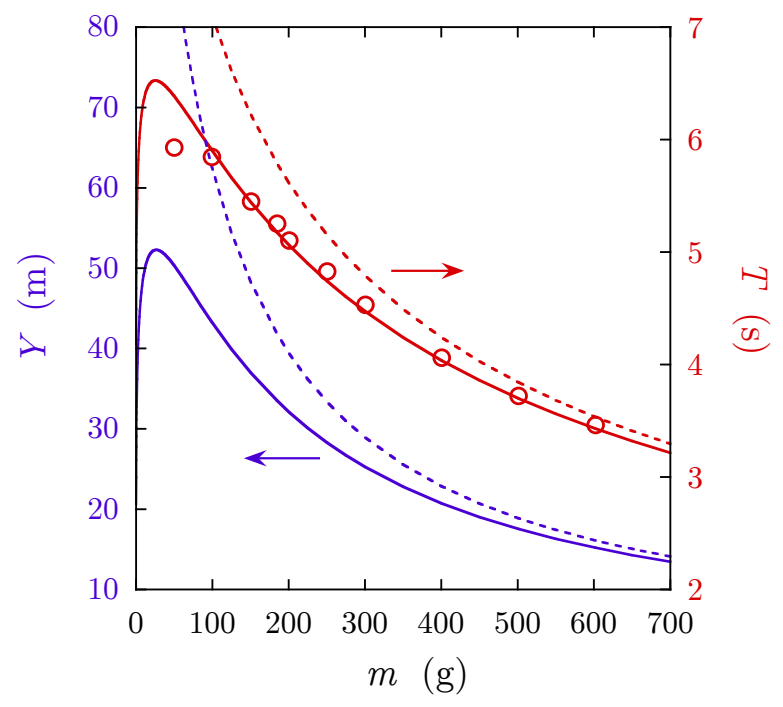

FIG. 7. For the right axis, measured times of flight $T$ in seconds for vertical launches vs. balloon mass $m$ in grams (circles) compared with Eq. (26) that includes drag (solid trace) and with Eq. (30) that excludes drag (dashed trace). For the left axis, the apex height $Y$ in meters for vertical launches vs. balloon mass $m$ in grams from Eq. (21) that includes drag (solid trace) and from Eq. (29) that excludes drag (dashed trace).

entire flight. The measured time of flight for the 50-g (smallest) balloon falls short of the prediction, indicating that the non-sphericity of this balloon, including the relatively large profile of its tied-off neck, might require a drag coefficient that is larger than 0.55 . The agreement between our measurements on flexible water balloons and our predictions for rigid spheres suggests that in-flight deformations of water balloons, including vibrations, might be negligible.

Also shown in Fig. 7 are the height $Y$ at apex given by Eq. (21) and the drag-free predictions of $Y$ and $T$ given by Eqs. (29) and (30), which indicate that drag plays a significant role for the smaller masses considered. Equations (21) and (26) predict that $Y \rightarrow y_{0}$ and $T \rightarrow \infty$ as $m \rightarrow 0$, implying that any initial speed quickly decays for a vanishingly small particle at the launch point, where the particle remains suspended for all time, consistent with the results $v_{t} \rightarrow 0$ and $\kappa \rightarrow \infty$ discussed previously [55].

Equation (16) enables us to correct the measurements of $v_{0}$ in Fig. 5 for air drag during the short $(D=3$ $\mathrm{m})$ horizontal flights. Neglecting the small gravitational deflection during this flight, Eq. (16) predicts that the speed of a horizontal projectile with initial speed $v_{0}$ decays to $v(D)=v_{0} e^{-\kappa D}$ over a distance $D$. Solving for $v_{0}$ in this equation and treating the measurements of $v_{0}$ in the previous section as $v(D)$ yields the corrected values plotted as filled circles in Fig. 5, thereby improving the agreement between the predicted and measured values of

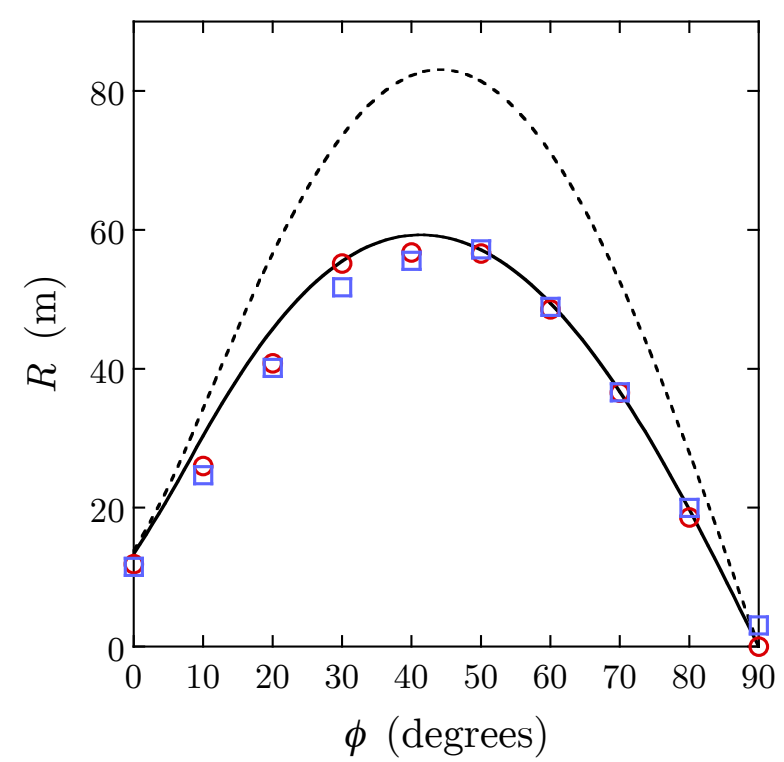

FIG. 8. Measurements of the horizontal range $R$ vs. launch angle $\phi$ for initial velocity toward the east (circles) and toward the west (squares), together with theoretical results for drag coefficient $C=0.55$ [solid trace, from numerical integration of Eq. (13)] and $C=0$ [dashed trace, Eq. (34)].

$v_{0}$. Corrections are larger for the smaller balloons, consistent with the arguments above that drag is important for small masses.

\section{RANGE}

Measuring the horizontal range $R$ vs. the launch angle $\phi$ follows the spirit of Angry Birds and allows beginning students to appreciate that two different values of $\phi$ can produce the same value of $R$. Using water balloons of mass $m=185 \pm 1 \mathrm{~g}$, we made two measurements at each launch angle, one with initial velocity to the east and the other to the west, with the balloons traversing the same length of ground from opposite directions (Fig. 8). During the 28 minutes required to take the measurements, the wind was light but variable, originating generally from the southeast with average wind speed $1.1 \pm 0.6$ $\mathrm{m} / \mathrm{s}$ and average direction of origin $140 \pm 60$ degrees, measured counterclockwise from north when viewed from above. The wind velocity measurements were taken with a Gill WindSonic Two-Dimensional Sonic Anemometer. We measured ranges $R$ from a point on the ground directly below the launcher axis to the center of the damp balloon landing site using a Kobalt 300-ft engineering tape measure. These damp landing sites showed clearly on the soil surface of the dry, uncultivated field.

For $C=0.55, \rho_{w}=1.0 \times 10^{3} \mathrm{~kg} / \mathrm{m}^{3}$, and $\rho_{a}=1.2$ $\mathrm{kg} / \mathrm{m}^{3}$, balloons of mass $m=185 \mathrm{~g}$ have radius $r=3.53$ cm from Eq. (15) and $\kappa=0.00701 \mathrm{~m}^{-1}$ from Eq. (14). 
The values $L_{0}=79 \mathrm{~cm}, L=193 \mathrm{~cm}, X=114 \mathrm{~cm}$, $M=0.02571 \mathrm{~kg}, \lambda_{0}=0.0427 \mathrm{~kg} / \mathrm{m}, a=0.56 \mathrm{~m}, b=1.23$ $\mathrm{m}, h=1.05 \mathrm{~m}, g=9.8 \mathrm{~m} / \mathrm{s}^{2}, U(X)=26.0 \mathrm{~J}$ from Eq. (9), $m_{k}=0.255 \mathrm{~kg}$ from Eq. (7), and $m_{p}=0.278 \mathrm{~kg}$ from Eq. (8) allow us to calculate the $\phi$-dependent initial coordinates (Fig. 2),

$$
\begin{gathered}
x_{0}(\phi)=a \cos \phi \\
y_{0}(\phi)=h+a \sin \phi,
\end{gathered}
$$

and the $\phi$-dependent initial speed [from Eq. (6)],

$$
v_{0}(\phi)=\left[\frac{8 U(X)}{m_{k}}-2 \frac{m_{p}}{m_{k}} g b \sin \phi\right]^{1 / 2},
$$

where the origin of coordinates is taken to be the point on the ground directly below the platform launcher axis.

Equation (13) governs the flight of the projectile starting with these initial conditions and ending at the landing point where $x=R$ and $y=0$. For $\kappa=0$, Eq. (13) reduces to the standard introductory physics problem of projectile motion with constant acceleration $\mathbf{g}$, yielding a range

$$
R=x_{0}+\frac{v_{0 x}}{g}\left(v_{0 y}+\sqrt{v_{0 y}^{2}+2 g y_{0}}\right)
$$

where $v_{0 x}=v_{0}(\phi) \cos \phi$ and $v_{0 y}=v_{0}(\phi) \sin \phi$. For $\kappa \geq 0$, Eq. (13) is easily integrated numerically using Euler's method. A time step of $0.001 \mathrm{~s}$ produces results that agree with Eq. (34) to within $0.1 \%$ for $\kappa=0$. We used a time step of $0.00001 \mathrm{~s}$, and double precision FORTRAN, to obtain the solid trace in Fig. 8. For vertical launches with drag, such Euler integration agrees with the predictions of Eq. (26) to within $0.02 \%$.

Figure 8 shows predictions for $C=0.55$ from numerical integration (solid trace), results for $C=0$ (dashed trace) from Eq. (34), and the measurements (data points). As might be expected, drag plays a smaller role in the range $R$ for near-horizontal $(\phi \approx 0)$ and near-vertical $\left(\phi \approx 90^{\circ}\right)$ launches than for intermediate angles. The corresponding values of $v_{0}$ given by Eq. (33) range from $28.1 \mathrm{~m} / \mathrm{s}$ for $\phi=90^{\circ}$ to $28.5 \mathrm{~m} / \mathrm{s}$ for $\phi=0$. The agreement between the predictions and measurements for large angles $\phi \geq 50^{\circ}$ and the agreement for the times of flight for vertical launches (Fig. 7) suggest that the observed shortfall in the measured values of $R$ for small angles may be due to a small amount of gravitational deflection during launch, deflection that is absent or negligible for vertical and near-vertical launches. For a vertical launch, the elastic force and gravity are antiparallel, and there is no gravitational deflection.

Including gravitational deflection for small launch angles would likely imply an initial velocity vector whose elevation angle $\theta$ is less than the launch platform elevation angle $\phi$. Lower elevation angles would decrease the predicted values of $R$ and might improve the small- $\phi$ agreement between theory and experiments in Fig. 8. Including this gravitational deflection would also introduce considerable complication, precluding the use of conservation of energy during the launch and rendering $x_{0}, y_{0}$, $v_{0}$, and $\theta$ dependent on time-dependent integrals over the elastic force. A study of this deflection might make an excellent senior thesis.

\section{CONCLUSIONS}

In this paper, we introduce a collapsible projectile launcher design that capitalizes on the popularity of Angry Birds to capture the interest of students in projectile motion, air drag, and nonlinear elastic energy. The design features a fully adjustable, reproducible initial velocity vector. Using water balloons as projectiles provides a convenient means of identifying the landing site (by a damp spot on the ground) and a convenient means for varying the projectile mass (by changing the volume of water in the balloon). For the experiments reported here, the slingshot handles were attached at the $100 \mathrm{~cm}$ mark discussed in Ref. [1]. Attaching these handles at the 120 $\mathrm{cm}$ mark yields ranges up to $85 \mathrm{~m}$ for smaller balloons.

The theory presented here, which includes the inertia of the slingshot bands and pouch, agrees well with experiments and suggests that gravitational deflection during launch may play an observable role for small launch angles. The launcher can be built and the experiments carried out using readily-available, inexpensive tools and materials, including an iPhone software application to make accurate time measurements. Analytical results are presented for rectilinear motion with quadratic drag, and simple Euler integration is used for two-dimensional motion with drag.

In addition to water balloons, the launcher can be used on softballs, baseballs, golf balls, and other small projectiles in order to study the range of balls with different masses, sizes, and Reynolds numbers. Launch speeds were determined by measuring the time taken to travel a fixed distance, and might alternatively be determined through video photography, by measuring the distance traveled between successive frames.

\section{ACKNOWLEDGEMENTS}

We gratefully acknowledge Danny Bourgeois, Nadine Edwards, Robert Edwards, Jordan Evans, Alyssa Sam, Kalyn Shorthill, and Kevin Woodward for technical assistance, Randy Anderson and Seth Lyman for assistance with wind velocity measurements, and W. Farrell Edwards for discussions about velocity measurements. Support from NSF Grant No. CBET 1332265 is gratefully acknowledged. 
[1] M. A. Christiansen, B. F. Edwards, and D. D. Sam, "Schematics of a Water Balloon Launcher Design and Reproducible Water-Balloon-Filling Procedures Used for a Middle School Summer Science Camp. USU Uintah Basin Faculty Publications 2013, Paper 1.

http://digitalcommons.usu.edu/ub_facpubs/1/ (accessed August 6, 2013).

[2] G. Liesik, "Kids learn science of Angry Birds at USU camp," KSL television story, June 4, 2012,

http://www.ksl. com/?nid=960\&sid=20662672 (accessed August 6, 2013).

[3] P. Nord, Water Balloon Launcher, http://youtu.be/93Q3axlxixk (accessed August 6, 2013).

[4] P. Nord, Balloon Launcher Quick-Release, http://youtu.be/DfYjYZdD6PU (accessed August 6, 2013).

[5] J. Gaudiosi (March 11, 2012), "Rovio Execs Explain What Angry Birds Toons Channel Opens Up To Its 1.7 Billion Gamers,"

http: //www . forbes.com/ (accessed August 6, 2013).

[6] "Angry Birds will be bigger than Mickey Mouse and Mario. Is there a success formula for apps?" MIT Entrepreneurship Review. February 18, 2011.

http://miter.mit.edu/

(accessed August 6, 2013).

[7] Akiyama, H.; Nishimura, J.; Namiki, M.; Okabe, Y.; Matsuzaka, Y.; Hirosawa, H. Advances in Space Research 3 1983, 97-100.

[8] E. E. Magnuson, One person missile-launching toy method, United States Patent No. 5,127,389, Issued July 7, 1992,

http://www. uspto.gov (accessed Aug 7, 2013).

[9] Trinity University Engineering Science/Design Sequence Homepage.

http://web.trinity.edu/x7494.xml (accessed Aug 7, 2013).

[10] Trinity University Balloon Launcher Project Homepage. http://www.resnet.trinity.edu/cbauer/page $\backslash \% 204 . h t m$ (accessed Aug 7, 2013).

[11] http://www.instructables.com/id/Water-Balloon/Launcher

(accessed Aug 7, 2013).

[12] B. Bullock, 400 Yard Waterballon Slingshot http://youtu.be/IqnicAK-cPc (accessed Aug. 7, 2013).

[13] H. Lamb, Hydrodynamics, Sixth Edition (Dover Publications, New York, 1993), Section 337, page 599.

[14] James Lock, "The physics of air resistance," The Physics Teacher, 20, 158-160 (1985).

[15] R. D. H. Warburton and J. Wang, "Analysis of asymptotic projectile motion with air resistance using the Lambert W function," Am. J. Phys. 72, 1404-1407 (2004).

[16] D. A. Morales, "A generalization on projectile motion with linear resistance," Can. J. Phys. 89, 1233-1250 (2011).

[17] H. Hu, Y. P. Zhao, Y. J. Guo, and M. Y. Zheng, "Analysis of linear resisted projectile motion using the Lambert $\mathrm{W}$ function," Acta Mech. 223, 441-447 (2011).
[18] The Engineering Toolbox, http://www.engineeringtoolbox.com/air-absolute-

kinematic-viscosity-d_601.html

(accessed August 20, 2013).

[19] P. Timmerman and J. P. van der Weele, "On the rise and fall of a ball with linear or quadratic drag," Am. J. Phys. 67, 538-546 (1999).

[20] R. Borghi, "Trajectory of a body in a resistant medium: An elementary derivation," Eur. J. Phys. 34, 359-369 (2013).

[21] G. W. Parker, "Projectile motion with air resistance quadratic in the speed," Am. J. Phys. 45, 606-610 (1977).

[22] Michael a. B. Deakin, "Approximate trajectories for projectile motion with air resistance," Am. J. Phys. 66, 3437 (1998).

[23] K. Yabushita, M. Yamashita, K. Tsuboi, "An analytic solution of projectile motion with the quadratic resistance law using the homotopy analysis method," J. Phys. A 40, 8403-8416 (2007).

[24] Peter J. Brancazio, "Trajectory of a fly ball," Phys. Teach. 23, 20-23 (1985).

[25] R. D. Mehta, Ann. Rev. Fluid Mech. 17, 151-189 (1985).

[26] Cliff Frohlich, "Resource Letter PS-2: Physics of Sports," Am. J. Phys. 79, 565-574 (2011).

[27] Joseph M. Zayas, "Experimental determination of the coefficient of drag of a tennis ball," Am. J. Phys. 54, 622-625 (1986).

[28] Ken Takahashi, "Measuring air resistance in a computerized laboratory," Am. J. Phys. 67, 709-711 (1999).

[29] Alan M. Nathan, "The effect of spin on the flight of a baseball," Am. J. Phys. 76, 119-124 (2008).

[30] Michael K. McBeath, Alan M. Nathan, a. Terry Bahill, and David G. Baldwin, "Paradoxical pop-ups: Why are they difficult to catch?," Am. J. Phys. 76, 723-729 (2008).

[31] J. E. Goff and M. J. Carre, "Trajectory analysis of a soccer ball," Am. J. Phys. 77, 1020-1027 (2009).

[32] Rod Cross, "Aerodynamics in the classroom and at the ball park," Am. J. Phys. 80, 289-297 (2012).

[33] T. Walley Williams, "Apparatus Drawings Project. Report Number 32. Launching Tube for a Laboratory Experiment on Projectile Motion," Am. J. Phys. 30, 851853 (1962).

[34] Richard M. Sutton, "Three Demonstration Experiments on Projectile Motion," Am. J. Phys. 12, 104-105 (1944).

[35] Vasilis Pagonis, David Guerra, Sean Chauduri, Brian Hornbecker, and Nathan Smith, "Effects of air resistance," Phys. Teach. 35, 364-368 (1997).

[36] J. Pantaleone and J. Messer, "The added mass of a spherical projectile," Am. J. Phys. 79, 1202-1210 (2011).

[37] Jorge Ballester and Chuck Pheatt, "Using the Xbox Kinect sensor for positional data acquisition," Am. J. Phys. 81, 71-77 (2013).

[38] Herman Erlichson, "Maximum projectile range with drag and lift, with particular application to golf," Am. J. Phys. 51, 357-362 (1983).

[39] C. W. Groetsch, "On the optimal angle of projection in general media," Am. J. Phys. 65, 797-799 (1997).

[40] Richard H. Price and J. D. Romano, "Aim high and go far? Optimal projectile launch angles greater than $45^{\circ}$," Am. J. Phys. 66, 109-113 (1998). 
[41] M. J. Carr, S. R. Goodwill, and S. J. Haake, "Understanding the effect of seams on the aerodynamics of an association football," J. Mech. Eng. Sci. 219, 657-666 (2005).

[42] T. Asai, K. Seo, O. Kobayashi, and R. Sakashita, "Fundamental aerodynamics of the soccer ball, Sports Eng. 10, 101-110 (2007).

[43] C. M. Romo-Kroger, "Testing plastic deformations of materials in the introductory undergraduate mechanics laboratory," Eur. J. Phys. 33, 551-563 (2012).

[44] C. A. Triana and F. Fajardo, "Dependence of some mechanical properties of elastic bands on the length and load time," Eur. J. Phys. 33, 771-784 (2012).

[45] David Roundy and Michael Rogers, "Exploring the thermodynamics of a rubber band," Am. J. Phys. 81, 20-23 (2013).

[46] Vyacheslavas Kashcheyevs, "Exact non-Hookean scaling of cylindrically bent elastic sheets and the largeamplitude pendulum," Am. J. Phys. 79, 657-661 (2011).

[47] Gareth J. Lewis, J. Cris Arnold, and Iwan W. Griffiths, "The dynamic behavior of squash balls," Am. J. Phys. 79, 291-296 (2011).

[48] Rod Cross, "Elastic and viscous properties of Silly Putty," Am. J. Phys. 80, 870-875 (2012).
[49] Rod Cross, "The bounce of a ball," Am. J. Phys. 67, 222-227 (1999).

[50] Drag on a Sphere, National Aeronautics and Space Administration, wWw.grc.nasa.gov/WWW/k-12/airplane/dragsphere.html (accessed August 20, 2013).

[51] R. D. Knight, Physics for Scientists and Engineers: A Strategic Approach, Third Edition, (Pearson, Glenview, IL, 2013) Sec. 6.5, p. 153.

[52] The range $3 \times 10^{2}<\operatorname{Re}<1.3 \times 10^{5}$ pertains to our Reynolds number $\operatorname{Re}=r v / \nu$ and corresponds to the range $6 \times 10^{2}<\operatorname{Re}^{\prime}<2.5 \times 10^{5}$ shown in Fig. 1 of Ref. [19], where the Reynolds number $\operatorname{Re}^{\prime}=d v / \nu$ is defined using the sphere diameter $d=2 r$.

[53] R. A. Becker, Introduction to Theoretical Mechanics (McGraw-Hill, New York, 1954), Sec. 6-8.

[54] The behavior $v_{t} \propto m^{1 / 6}$ results from the quadratic drag force of Eq. (12). Linear Stokes drag governs very small$m$ behavior, $v_{t} \propto r^{2} \propto m^{2 / 3}$, which pertains to $\operatorname{Re}<1$ and microscopic particles with radii $r<2 \times 10^{-5} \mathrm{~m}$ [13]. Both quadratic and linear drag, and both forms of $v_{t}$, predict suspension of vanishingly small particles.

[55] The $T \rightarrow \infty$ behavior as $m \rightarrow 0$ predicted by Eq. (26) is not reflected in Fig. 7 because the approach to infinity begins at microscopic masses $m<10^{-6} \mathrm{~g}$ that are not shown in the figure. See [54]. 